\title{
Using nonhuman culture in conservation requires careful and concerted action
}

\author{
Susana Carvalho ${ }^{1,2,3} \quad$ | Erin G. Wessling ${ }^{4}$ (c) | Ekwoge E. Abwe ${ }^{5,6}$ \\ Katarina Almeida-Warren ${ }^{1,3}$ (6) | Mimi Arandjelovic ${ }^{7}$ | Christophe Boesch ${ }^{7,8}$ \\ Emmanuel Danquah $^{9}$ | Mamadou Saliou Diallo ${ }^{10}$ | Catherine Hobaiter ${ }^{11}$ (] | \\ Kimberley Hockings ${ }^{12} \quad$ | Tatyana Humle ${ }^{13} \quad$ Rachel Ashegbofe Ikemeh ${ }^{14}$ | \\ Ammie K. Kalan ${ }^{15}$ | Lydia Luncz $^{16}$ | Gaku Ohashi ${ }^{17}$ |
}

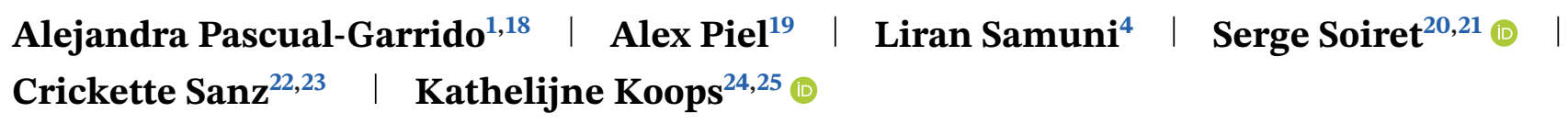

${ }^{1}$ Primate Models for Behavioural Evolution Lab, Institute of Human Sciences, University of Oxford, Oxford, UK

${ }^{2}$ Gorongosa National Park, Sofala, Mozambique

${ }^{3}$ Interdisciplinary Center for Archaeology and Evolution of Human Behaviour, Universidade do Algarve, Faro, Portugal

${ }^{4}$ Department of Human Evolutionary Biology, Harvard University, Cambridge, Massachusetts

${ }^{5}$ San Diego Zoo Wildlife Alliance, San Diego, California

${ }^{6}$ Ebo Forest Research Project, Douala, Cameroon

${ }^{7}$ Max Planck Institute for Evolutionary Anthropology, Leipzig, Germany

${ }^{8}$ Wild Chimpanzee Foundation, Cologny, Switzerland

${ }^{9}$ Faculty of Renewable Natural Resources, Department of Wildlife and Range Management, Kwame Nkrumah University of Science and Technology, Kumasi, Ghana

${ }^{10}$ Guinée Ecologie, Conakry, Guinea

${ }^{11}$ School of Psychology and Neuroscience, University of St Andrews, St Andrews, UK

${ }^{12}$ Centre for Ecology and Conservation, University of Exeter, Penryn, UK

${ }^{13}$ Division of Human and Social Science, School of Anthropology and Conservation, Durrell Institute of Conservation and Ecology, University of Kent, Canterbury, UK

${ }^{14}$ SW/Niger Delta Forest Project, Garki, Abuja, Nigeria

${ }^{15}$ Department of Anthropology, University of Victoria, Victoria, Canada

${ }^{16}$ Technological Primates Research Group, Max Planck Institute for Evolutionary Anthropology, Leipzig, Germany

${ }^{17}$ Chubu University, Kasugai, Aichi, Japan

${ }^{18}$ Department of Anthropology, Durham University, Durham, UK

${ }^{19}$ Department of Anthropology, University College London, London, UK

${ }^{20}$ Laboratoire de Zoologie et de Biologie Animale, Université Félix Houphouët-Boigny, Abidjan, Côte d'Ivoire

${ }^{21}$ Centre Suisse de Recherches Scientifiques en Côte d'Ivoire (CSRS), Abidjan, Côte d'Ivoire

22 Department of Anthropology, Washington University in Saint Louis, Saint Louis, Missouri

${ }^{23}$ Wildlife Conservation Society, Congo Program, Brazzaville, Republic of Congo

${ }^{24}$ Ape Behaviour \& Ecology Group, Department of Anthropology, University of Zurich, Zürich, Switzerland

${ }^{25}$ Department of Archaeology, University of Cambridge, Cambridge, UK 


\section{Correspondence}

Susana Carvalho, Primate Models for Behavioural Evolution Lab, Institute of Human Sciences, University of Oxford, Oxford, UK.

Email:susana.carvalho@anthro.ox.ac.uk Erin G. Wessling, Department of Human Evolutionary Biology, Harvard University, Cambridge, MA.

Email: ewessling@fas.harvard.edu Crickette Sanz, Department of Anthropology, Washington University in Saint Louis, MO 63130.

Email:csanz@wustl.edu

Kathelijne Koops, Ape Behaviour \& Ecology Group, Department of Anthropology, University of Zurich, Zürich, Switzerland. Email: kathelijne.koops@uzh.ch

Susana Carvalho, Erin G. Wessling, Crickette Sanz, and Kathelijne Koops contributed equally.

\begin{abstract}
Discussions of how animal culture can aid the conservation crisis are burgeoning. As scientists and conservationists working to protect endangered species, we call for reflection on how the culture concept may be applied in practice. Here, we discuss both the potential benefits and potential shortcomings of applying the animal culture concept, and propose a set of achievable milestones that will help guide and ensure its effective integration existing conservation frameworks, such as Adaptive Management cycles or Open Standards.
\end{abstract}

\section{KEYWORDS}

Adaptive Management, animal culture, conservation policy, Open Standards, target definition

\section{1 | INTRODUCTION}

Culture was long thought to be unique to humans, but has since been identified in a growing number of taxa (Whiten, 2021). Nonhuman culture continues to stimulate novel research and extensive debate. The most recent examples of its relevance and research scope concern the importance of documenting and preserving nonhuman cultural heritage for future generations (Kühl et al., 2019), as well as the potential role of animal culture in biodiversity conservation such as considering social learning and culture as conservation units (Brakes et al., 2019, 2021). More specifically, the integration of animal culture into conservation applications has been proposed as a means to maximize the survival prospects and reproductive outputs of individuals, social groups, and populations. One example is the argument that certain individuals within a social group may require targeted protection as important repositories of knowledge (e.g., elephant matriarchs; Brakes et al., 2019, 2021). Moreover, a focus on culture may help attract public interest (i.e., culture as a trait that connects humans to nonhuman species) and may promote funding for and focus on flagship species. But, the proposed use of nonhuman culture as a conservation tool leads to the critical question of: how can an animal culture approach be practically applied to enhance species conservation? More importantly, how can we ensure that empirical data on behavioral variation are fully integrated into applied, costefficient, and sustainable, conservation practices?

Currently, conservation prioritization practices focus on the most vulnerable species and populations, and on areas with high biodiversity. The current wave of "culture for conservation" arguments call for a more holistic assessment of individual, population, and species viability, which goes beyond population statistics to include the specific behavioral and ecological contexts crucial for population maintenance and survival. While "culture for conservation" can aid in bridging this gap, we suggest it should not be used as a stand-alone conservation tool. Rather, the culture concept must be integrated within existing conservation strategies, using an approach that is rigorous and evidence-based while also being attainable and efficient to implement. The culture for conservation concept should both complement and enhance current conservation standards (Junker et al., 2020). Given the urgent need for actionable and tractable responses to the alarming biodiversity decline, we call for caution in how the concept of culture is applied in conservation practice, and suggest milestones to develop a monitorable and effective approach to the use of animal culture as a conservation tool that accounts for (and balances) its risks and benefits.

\section{2 | FROM THEORY TO PRACTICE}

For many, the notion of using animal culture in conservation still remains abstract and a practical pathway is needed to ensure the culture concept is effectively applied. To advance the discussion of how animal culture can be used in conservation, we propose that specific milestones be met before further action in a particular species or population is undertaken (Figure 1). Most of these 


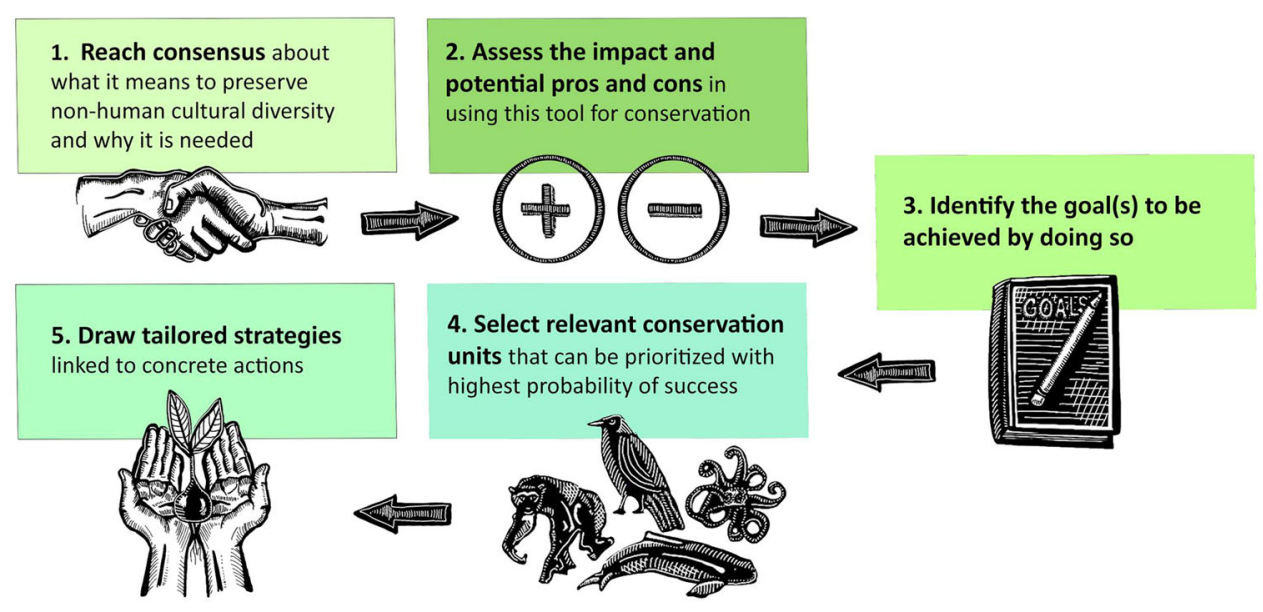

F I G U R E 1 Milestones for an effective incorporation of nonhuman cultures into conservation planning. Specific actions and outputs for the different milestones could include stakeholder workshops to generate guidelines on preserving cultural diversity, impact assessments based on pilot projects and studies, integrating goals into action plans, a priority list of conservation units, and planning research and conservation projects

milestones mirror common conservation practices such as the Open Standards approach (CMP, 2020), in that they aim to establish tangible goals and facilitate comprehensive solutions to a holistic perspective of threats to conservation targets. For an effective process, the definition of these milestones should be guided by a diverse and representative group of stakeholders, for example, experts, conservationists, governmental officials, local communities, and NGOs. Due to structural similarities to these approaches, the proposed process can be incorporated into existing conservation frameworks, such as Adaptive Management (McCarthy \& Possingham, 2007) or Open Standards (CMP, 2020).

First, conservationists and scientists would benefit from reaching an operational consensus on fundamental concepts and criteria for what constitutes animal culture in the wild, what it means to preserve it, and why culture preservation is needed. This exercise (see Point 1, Figure 1) must be realistic and should not hinder conservation efforts, but is necessary to determine which conservation units or practices are best suited to apply this new tool. Operationalizing the concepts of culture and behavioral diversity will help determine (1) whether all species that exhibit social learning should be primary "conservation through culture" targets and/or (2) whether efforts should prioritize species with an established cultural repertoire, that is, a set of group-specific, socially transmitted behaviors (e.g., orangutans, Pongo spp.; or killer whales, Orcinus orca).

Second, once we have reached consensus on the need for invoking the culture concept and an operational definition of culture as it relates to conservation is formalized, assessments based on pilot projects and ongoing field research can be used to better understand the potential impacts (positive and negative) of using culture as a tool for conservation (see Point 2, Figure 1).

Next, we need to identify the goals to be achieved by using culture in conservation (see Point 3, Figure 1), develop prioritization of these targets (if multiple), and agree on actionable processes. Such an approach is similar to the definition of goal formation (i.e., conservation target and vision definition) during processes like Open Standards (CMP, 2020). After goals are defined, the identification of requisite information for effective practice will necessarily follow and become clear. For example, conservationists may need to adopt strategies to accommodate understudied species or populations where knowledge of their behavioral and cultural repertoire is currently unknown or incomplete, but this can only be achieved once a shared understanding of what constitutes culture is established.

As a next milestone, we can proceed to select relevant conservation units that adhere to the definition of our conservation goals and can be prioritized with the highest probability of success (see Point 4, Figure 1). A practical example would be the need to decide whether species that have large-scale, socially learned, migratory routes, such as right whales (Eubalaena australis) should fall under the same "culture for conservation" strategy as species that have limited (albeit trans-national) ranging patterns but display diverse behavioral and cultural repertoires, such as chimpanzees (Pan troglodytes), or New Caledonian crows (Corvus moneduloides).

Finally, it is necessary to draw tailored, context-specific strategies linked to concrete actions (see Point 5, Figure 1) in order to fully move from theory to conservation practice. These actions will involve monitoring of effectiveness 
of proposed action to conserving the selected conservation units.

While the proposed milestones provide a practical means to adapt culture theory into conservation practice, there are some additional concerns that practitioners must consider. For example, social structure and pathways of social information transfer are important for assessing cultural attributes, but are also interconnected with wellestablished conservation metrics such as population size, viability, and demography. Given the use of concepts such as flagship, umbrella, or keystone species when advocating for conservation of various species, can cultural attributes of a nonhuman species aid in promoting a species' status, or would these be redundant to already existing arguments for conservation? There is potential value in using nonhuman culture to attract public interest and elicit empathy toward species of concern, but this requires sensitive and tailored messaging for different audiences and contexts. Abuse of this approach could lead to allocation of limited conservation resources based on a unidimensional metric, for example, culture.

\section{3 | FILLING GAPS AND AVOIDING PITFALLS}

To highlight some important gaps in the current discussions of "culture for conservation," we refer to the wellstudied and often-cited example of nut cracking by wild chimpanzees (Brakes et al., 2019, 2021). Nut cracking is proposed as a single behavior of key conservation concern due to a suggested link to vital rates, such as reproduction and survival (Brakes et al., 2021). However, it could be argued that populations with high behavioral diversity may be more resilient to environmental change (Kalan et al., 2020; Whitehead \& Rendell, 2015). Before applying the concept of culture to conservation practice, practitioners must reach a consensus about the conservation value of single behaviors (e.g., nut cracking) compared to overall behavioral diversity in species conservation.

For chimpanzees, how do non-nut cracking, but behaviorally diverse populations fit into conservation-throughculture schemes? The answer depends on how conservation policy makers prioritize the intrinsic value and cultural indicators of populations, independent of their influence on vital rates. We argue that cultural behavior need not necessarily be linked to vital rates, as previously proposed (Brakes et al., 2021) in order to be prioritized. Moreover, it is unclear whether cultural foraging behaviors like nut cracking are comparatively more important for the maintenance of vital rates than hygiene, comfort, or selfmedication cultural behaviors. A sole focus on vital rates raises difficulties for practitioners regarding how to incor- porate cultural variation in social behaviors or communication (e.g., the grooming hand-clasp or group dialects in chimpanzees), into conservation responses. In these cases, the link from culture to survival and reproductive rates may be indirect at best, although these behaviors may play a significant role in social cohesion and grouping patterns. Even for a behavior like nut cracking, which is often proposed to be a response to food scarcity and thus may be more intuitively linked to vital rates than nonforaging behaviors (e.g., Yamakoshi, 1998), the proposed relationship with food scarcity remains empirically inconsistent (Koops et al., 2014). Despite being one of the most studied tool-use behaviors of one of the most intensively studied animals, a clear answer as to the influences of nut cracking on reproduction and survival is still lacking.

Arguments for conservation, therefore, cannot be contingent on meeting (impractically) high empirical standards, such as a link to vital rates (Brakes et al., 2019, 2021). Specifically, behaviors like nut cracking serve as a demonstration of why the long-term research needed to establish these connections is incompatible with the urgent timeframe for which we must act. Consequently, adding cultural uniqueness as a criterion for conservation efforts might lead to delay in taking conservation action if these links must first be demonstrated. Importantly, it may be impossible for a nonhuman animal (in practice) to meet the largely academic cultural benchmarks set by recent proposals (Brakes et al., 2019, 2021). Proposed benchmarks such as "understanding linkages between culture and vital rates, cultural evolution, and adaption to rapid global change" or "understand[ing] the circumstances under which social learning and culture are likely to impact population viability through phenotypic variation" (Brakes et al., 2021, P. 8) are practically challenging, and are unlikely to translate into effective conservation practices in a short time frame. We therefore caution that advancing weak evidence and proposing unclear benchmarks has the potential to undermine both the uptake, as well as the utility, of culture as a criterion for conservation effort altogether.

It follows that a focus on such benchmarks could reduce credibility of the culture concept to local stakeholders who play a critical role in ensuring sustainable conservation on the ground. For example, a lack of clear definitions about what constitutes animal culture or sufficient evidence for self-defined necessary benchmarks (e.g., social transmission; Brakes et al., 2019, 2021) may lead to ineffective communication with policy makers, government officials, and conservation NGOs. To advance conservation initiatives for endangered species, immediate messaging of how such a framework can be applied is needed because species often listed as key examples of cultural species are in free-fall. The nut cracking western chimpanzees (P. $t$. 
verus) are critically endangered and have declined by $80 \%$ over the last two decades (Kühl et al., 2017). Whooping cranes (Grus americana), which are observed to have social learning, are down to a relictual population in the wild (Mueller et al., 2013). Given the severity of losses in the face of growing anthropogenic threats, scholars and practitioners alike need to be aware of the consequences of proposed but insufficiently defined solutions that risk delaying effective action, and should seek to mitigate those consequences as best as possible.

\section{4 | TOWARD CAREFUL AND CONCERTED ACTION}

Scientists and conservationists must be aware of the pitfall of prioritizing the monitoring of extinction crises rather than taking steps toward mitigating them (Lindenmayer et al., 2013; Nichols \& Williams, 2006). Whereas data gathering may provide insights into the direct benefits of cultural behaviors to conservation, or help identify new cultural traits across populations, encouraging further efforts to fill remaining knowledge gaps may be misguided when conservation needs are immediate and financial support is limited. Adoption of culture as a conservation tool could potentially help garner broader support for conservation efforts, but it could also imply a trade-off, where a finite amount of effort is distributed among a larger number of populations. There is a risk that the number of populations considered conservation priorities increases significantly if criteria for prioritization are not clearly defined and rapidly measurable. Discoveries of new cultural traits could be boundless and may continuously grow with each new studied population (Boesch et al., 2020). Large-scale cultural surveying necessitates immense effort, funding, and crucially, time, which may detract from direct conservation actions. Importantly, the discovery of new cultural traits will not provide further justification for the argument that cultural heritage of nonhuman species must be preserved in species where it is already established. To develop effective conservation strategies, researchers studying animal cultures and conservation practitioners need to remain in close communication to ensure efforts in both disciplines are neither wasted nor duplicated, and remain focused toward tangible, well-defined goals.

We must also consider how the ability for a given species or population to meet the benchmarks to be classified as a "cultural species" may impart cascading biases on how conservation need is identified and how action is subsequently prioritized. Knowledge of the social-learning mechanisms of behavioral traits are already biased toward well-studied populations of well-known species (Besanson
\& McNamara, 2019). Such populations are most likely to be the first groups for which benchmarks, such as culturedependent viability, can be reached, while also already being among the best protected (Besanson \& McNamara, 2019). Additionally, this bias extends to how detectable or identifiable traces of cultural behaviors may be. While some behaviors, like tool use, are more easily quantifiable and can be recorded based on indirect evidence, other behaviors, like communicative signals, require years of direct observation to thoroughly understand. In that sense, the observable behavioral diversity across populations is functionally biased toward material cultures. Hence, we need to tailor our approach both to the species/populations and to the types of cultural behaviors recorded.

\section{5 | CONCLUSION}

While a goal of conservation action is often to conserve particular species, it can also be about maintaining, restoring, and enhancing ecosystem function and services, and protecting biological diversity as a whole-irrespective of a species' status as cultural or not. To address this, connections between animal cultures and habitat protection need to be clarified. The core aim of future efforts should be to directly contribute to conservation, which may involve initiatives like formalized action planning (IUCN, 2020), awareness raising, community-based conservation, and local human development. Moreover, conserving nonhuman cultural heritage for future generations should also include documentation, curation, and preservation of material (and nonmaterial) records. Advocacy for incorporation of nonhuman culture into conservation must ultimately complement, and not compete with, existing activities and tools. Following the proposition of formal, and clear milestones (Figure 1), we can best contribute to the shared goals of conservation by developing a multilevel approach that transcends both scale and actor, and includes leadership by local communities, connects with local human development projects, considers a landscape approach to conservation, and promotes global awareness.

\section{ACKNOWLEDGMENTS}

This manuscript was improved by feedback from several anonymous reviewers. We thank Elodie Freymann and João Coelho for assistance with graphics.

\section{AUTHOR CONTRIBUTIONS}

SC, EW, CS, and KK conceptualized and drafted the manuscript. All authors conceptualized and revised the manuscript, and agreed to be held accountable for the content therein. 


\section{DATA ACCESSIBILITY STATEMENT}

This manuscript presents no primary data.

\section{CONFLICT OF INTEREST}

The authors declare no conflicts of interest.

\section{O R C I D}

Erin G. Wessling (1) https://orcid.org/0000-0001-9661-4354

Katarina Almeida-Warren (1) https://orcid.org/0000-0002-

7634-9466

Catherine Hobaiter (10) https://orcid.org/0000-0002-3893-

0524

Serge Soiret 10 https://orcid.org/0000-0001-6773-500X

Kathelijne Koops (10) https://orcid.org/0000-0001-7097-2698

\section{REFERENCES}

Bezanson, M., \& McNamara, A. (2019). The what and where of primate field research may be failing primate conservation. Evolutionary Anthropology: Issues, News, and Reviews, 28, 166-178.

Boesch, C., Kalan, A. K., Mundry, R., Arandjelovic, M., Pika, S., Dieguez, P., Ayimisin, E. A., Barciela, A., Coupland, C., Egbe, V. E., Eno-Nku, M., Fay, J. M., Fine, D., Hernandez-Aguilar, R. A., Hermans, V., Kadam, P., Kambi, M., Llana, M., Maretti, G.,... Kühl, H. S. (2020). Chimpanzee ethnography reveals unexpected cultural diversity. Nature Human Behaviour, 4, 910-916.

Brakes, P., Dall, S. R. X., Aplin, L. M., Bearhop, S., Carroll, E. L., Ciucci, P., Fishlock, V., Ford, J. K. B., Garland, E. C., Keith, S. A., McGregor, P. K., Mesnick, S. L., Noad, M. J., di Sciara, G. N., Robbins, M. M., Simmonds, M. P., Spina, F., Thornton, A., Wade, P. R.,...Rutz, C. (2019). Animal cultures matter for conservation. Science, 363(6431), 1032-1034. https://doi.org/10.1126/ science.aaw3557.

Brakes, P., Carroll, E. L., Dall, S. R. X., Keith, S. A., McGregor, P. K., Mesnick, S. L., Noad M. J., Rendell L., Robbins M. M., Rutz C., Thornton A., Whiten A., Whiting M. J., Aplin, L. M., Bearhop S., Ciucci P., Fishlock V., Ford J. K. B., Notarbartolo di Sciara G,... Garland, E. C. (2021). A deepening understanding of animal culture suggests lessons for conservation. Proceedings of the Royal Society B: Biological Sciences, 288, rspb.2020.2718.

Conservation Measures Partnership (CMP). (2020). Open standards for the practice of conservation. Version 4.0.

IUCN. (2020). Regional action plan for the conservation of western chimpanzees (Pan troglodytes verus) 2020-2030. Regional action plan for the conservation of western chimpanzees (Pan troglodytes verus) 2020-2030. IUCN, International Union for Conservation of Nature. https://doi.org/10.2305/IUCN.CH.2020.SSC-RAP.2.en

Junker, J., Petrovan, S. O., Arroyo-Rodríguez, V., Boonratana, R., Byler, D., Chapman, C. A., \& Kühl, H. S. (2020). A severe lack of evidence limits effective conservation of the world's primates. Bioscience, 70, 794-803.
Kalan, A. K., Kulik, L., Arandjelovic, M., Boesch, C., Haas, F., Dieguez, P., Barratt, C. D., Abwe, E. E., Agbor, A., Angedakin, S., Aubert, F., Ayimisin E. A., Bailey, E., Bessone, M., Brazzola, G., Buh, V. E., Chancellor, R., Cohen, H., Coupland, C.,...Kühl, H. S. (2020). Environmental variability supports chimpanzee behavioural diversity. Nature Communications, 11, 4451.

Koops, K., Visalberghi, E., \& van Schaik, C. P. (2014). The ecology of primate material culture. Biology Letters, 10, 20140508.

Kühl, H. S., Boesch, C., Kulik, L., Haas, F., Arandjelovic, M., Dieguez, P., Bocksberger, G., Agbor, A., Angedakin, S., Ayimisin, E. A., Bessone, M., Brazzola, G., Chancellor, R., Cohen, H., Coupland, C., Danquah, E., Deschner, T., Dowd, D., Goedmakers, A.,... Kalan, A. K. (June 14, 2019). Inclusive chimpanzee conservation response. Science. American Association for the Advancement of Science.

Kühl, H. S., Sop, T., Williamson, E. A., Mundry, R., Brugière, D., Campbell, G., Cohen, H., Danquah, E., Ginn, L., Herbinger, I., Jones, S., Junker, J., Kormos, R., Kouakou, C. Y., N'Goran, P. K., Normand, E., Shutt-Phillips, K., Tickle, A., Vendras, E.,... Boesch, C. (2017). The critically endangered western chimpanzee declines by $80 \%$. American Journal of Primatology, 79, e22681.

Lindenmayer, D. B., Piggott, M. P., \& Wintle, B. A. (2013). Counting the books while the library burns: Why conservation monitoring programs need a plan for action. Frontiers in Ecology and the Environment, 11, 549-555.

McCarthy, M. A., \& Possingham, H. P. (2007). Active adaptive management for conservation. Conservation Biology, 21, 956-963.

Mueller, T., O’Hara, R. B., Converse, S. J., Urbanek, R. P., \& Fagan, W. F. (2013). Social learning of migratory performance. Science, 341, 999-1002.

Nichols, J. D., \& Williams, B. K. (2006). Monitoring for conservation. Trends in Ecology \& Evolution, 21, 668-673.

Whitehead, H., \& Rendell, L. (2015). The cultural lives of whales and dolphins. Chicago.

Whiten, A. (2021). The burgeoning reach of animal culture. Science, 372 , eabe6514.

Yamakoshi, G. (1998). Dietary responses to fruit scarcity of wild chimpanzees at Bossou, Guinea: Possible implications for ecological importance of tool use. American Journal of Physical Anthropology, 106, 283-295.

How to cite this article: Carvalho S., Wessling E. G., Abwe E. E., e al., Using nonhuman culture in conservation requires careful and concerted action. Conservation Letters. 2022;e12860. https://doi.org/10.1111/conl.12860 